\title{
Reconstrucción mandibular con combinación de injertos autólogos libres e instalación de implantes dentales: Reporte de Caso
}

Ventura-Ponce $H^{1}$, Cabrera $E^{3}$, Delgado-Azañero $W^{4}$, Huamani-Parra $J^{1}$, Ccahuana-Vasquez $V^{2}$, Lozano-Aquije $W^{3}$, Gárate $A^{5}$. Reconstrucción mandibular con combinación de injertos autólogos libres e instalación de implantes dentales: Reporte de Caso. Rev Estomatol Herediana. 2012 OctDic;22(4):216-22.

\section{RESUMEN.}

Entre los tumores mandibulares, destacamos el ameloblastoma como una patología frecuente en el área maxilofacial, descrito como un crecimiento local persistente, agresivo e invasivo con potencial de recidiva considerable, por lo que la integración clínica, imaginológica e histopatológica nos dirige a establecer diagnósticos para definir el tratamiento quirúrgico. Con el conocimiento de técnicas quirúrgicas maxilofaciales, indicadas para la ablación de tumores en el Maxilar inferior empleamos la mandibulectomía parcial o segmentaria con reconstrucción inmediata y posterior rehabilitación con implantes, demostrando efectividad en el tratamiento. La combinación de injertos autólogos ofrece: contención y estabilidad que da la cortical externa de calota craneana hacia el particulado de cresta ilíaca, y el volúmen óseo obtenido es brindado por el injerto particulado para facilitar la instalación de implantes.

Palabras claves: RECONSTRUCCIÓN MANDIBULAR, AMELOBLASTOMA, BIOPRÓTESIS IMPLANTES DENTALES.

Mandibular Reconstruction with autologous grafts combination and installation of dental implants: Case report.

\section{ABSTRACT}

Among the tumors mandibular ameloblastoma highlighted as a common pathology in the maxillofacial area, described as a persistent local growth, aggressive and invasive with significant potential for recurrence, so that clinical integration, imagiologic and histopathologic diagnoses directs us to define surgical treatment. With knowledge of maxillofacial surgical techniques, indicated for ablation of tumors in the lower jaw used the partial or segmental mandibulectomy with immediate reconstruction with implants and subsequent rehabilitation, demonstrating effectiveness in treatment. The combination of autologous grafts provides: containment and stability which gives the particulate calvaria into the iliac crest bone volume obtaining suitable for the installation of the implant provided by particulate graft.

Key words: MANDIBULAR RECONSTRUCTION, AMELOBLASTOMA, BIOPROSTHESIS, DENTAL IMPLANTS.

\section{Introducción}

Los tumores mandibulares frecuentes en la consulta del Cirujano Oral y Maxilofacial se destacan, los tumores odontogénicos queratoquísticos y el ameloblastoma describiendo a este último como una patología que muestra en su comportamiento una amplia agresividad y elevado potencial de recidiva de acuerdo a las diferentes formas de presentación histopatológica.

Los Ameloblastomas son tumores odontogénicos de origen epitelial, derivados principalmente del epitelio relacionado con la formación de la estructura dental; Restos celulares del Órgano del Esmalte (Malassez y Serrez),(1) Epitelio de Quistes Odontogénicos (Dentígeros y Odontomas), Alteraciones del Órgano del Esmalte en desarrollo, Células Basales del Epitelio de los Maxilares, desconociendo los factores desencadenantes para que se produzca la transformación neoplásica de dichas estructuras (2).

Epidemiológicamente, representa el $11 \%$ de los tumores odontógenos aproximadamente, y el $0.14 \%$

\section{Helard Ventura Ponce ${ }^{1}$ Edgar Cabrera Gómez ${ }^{3}$ Wilson Delgado Azañero ${ }^{4}$ Jaime Huamaní Parra ${ }^{1}$ Vanessa Ccahuana Vásquez ${ }^{2}$ Walter Lozano Aquije ${ }^{3}$ Arturo Gárate Arias ${ }^{5}$}

Especialista en Cirugía Oral y Maxilofacial. Docente del Departamento Académico de Medicina y Cirugía Buco Maxilofacial. Facultad de Estomatología Roberto Beltrán. Universidad Peruana Cayetano Heredia. Lima Perú.

Especialista en Rehabilitación Oral.

Residentes de Cirugía Oral y Maxilofacial de la UPCH.

Profesor Emérito. Profesor extraordinario investigador. Facultad de Estomatología Roberto Beltrán. Universidad Peruana Cayetano Heredia. Lima, Perú.

Residente de Implantología Oral.

\section{Correspondencia:}

Helard Ricardo Ventura Ponce.

Facultad de Estomatología Universidad

Peruana Cayetano Heredia.

Av. Honorio Delgado 350 Urb. Ingeniería

S.M.P Lima 31, Perú

Teléfono 3811950- 38111955 anexo 252 Lima-Perú.

E-mail: helard.ventura@upch.pe de todas las neoplasias. Se manifiesta prevalentemente durante la tercera, cuarta y quinta década de vida, sin predilección por el género, entre los 40 y 50 años de edad. Sin embargo la lesión se puede encontrar en cualquier grupo etario, afectando a niños y adolescentes $(1,2)$. En la revisión realizada por Zhang y colaboradores, 91,9\% de Ameloblastomas reportados en su serie fueron presentados en pacientes adolescentes en un grupo etario $\left(11^{\mathrm{a}}-20^{\mathrm{a}}\right)$, y el $8 \%$ restante en pacientes pediátricos cuyo rango se encontraba por debajo de los 
11 años (1), su localización muestra predilección cercana al $80 \%$ por el Hueso Mandibular (Región Cuerpo, Ángulo y Rama Mandibular), frecuentemente asociado a piezas dentarias no erupcionadas (4-10). El 20\% restante se ubican en hueso maxilar, caracterizándose por un peor pronóstico, ya que presentan un mayor índice de recidivas y potencial de malignización, pudiendo llegar a infiltrar el seno maxilar, la fosa pterigopalatina, la fosa temporal y diseminarse hacia las fosas nasales, órbitas o base de cráneo (2).

Se localiza con más frecuencia en la mandíbula con respecto al maxilar: el ángulo y la rama mandibular junto al área de los terceros molares inferiores representan las localizaciones más afectadas (1). Se han descrito numerosas variedades histológicas, algunas demuestran un solo subtipo histológico; otras se asocian dentro de una misma lesión (2)(3). En la actualidad existen diversas propuestas de tratamiento según la filosofía quirúrgica del cirujano, básicamente la conservadora y la radical.

Sus signos y síntomas varían de un paciente a otro, y en estadíos tempranos sólo consideramos hallazgos imaginológicos, Steven (4) describe que los Ameloblastomas mandibulares son detectados en un rango de 12 años antes que los hallados en los maxilares. El examen diagnóstico estándar es la radiografía panorámica, mientras que una Tomografía Cone-Beam está indicada en la fase de planificación quirúrgica (2). Su apariencia imaginológica varía en forma que se decriben como radiolucencias uniquísticas o multiquísticas con patrones de "Pompas de Jabón (Grandes) o Panal de Abeja (Pequeñas)" (5). La multiloculación caracteriza a las lesiones de mayor tamaño, la reabsorción de la raíz es rara, pero se observa, a veces en algunas lesiones de crecimiento rápido.

El tumor es de crecimiento lento, agresivo localmente y capaz de causar grandes deformidades faciales. La experiencia de años recientes ha mostrado que no todas las lesiones con características histológicas de ameloblastoma, tienen el mismo potencial de destrucción, recidiva e incluso metástasis. A medida que se acumula mayor información, resulta que a efectos terapéuticos, no todos los ameloblastomas exigen el mismo tratamiento quirúrgico, sino que es necesaria una correlación de rasgos clínicos, radiográficos e histológicos de cada lesión individual para determinar el subtipo clínico (6). Destacando la avidez en dicho tratamiento para contrarrestar las tasas de recurrencia considerables presentadas por este tipo de tumores (7).

Uno de los principales objetivos de la Reconstrucción Mandibular es sustituir el tejido duro y blando perdido en un sólo acto quirúrgico, garantizando la rápida recuperación de la zona afectada y de la zona donadora de dónde tomemos el injerto autólogo, con el mínimo de complicaciones existentes y logrando obtener una armonía facial en nuestros pacientes (18). Variedad de métodos y técnicas han sido reportadas en la literatura para conseguir la restauración de la continuidad mandibular deseada, entre los que se destacan: injertos libres microvascularizados de peroné y cresta ilíaca, injertos autólogos de calota craneana, cresta ilíaca, condro-costales, entre otros. La elección se basa en función al tamaño y volumen del defecto a reconstruir.

Con el reporte se destaca la importancia que le otorga los Ciruja- nos Orales y Maxilofaciales a la reconstrucción mandibular inmediata luego de la exceresis del tumor, realizando la combinación de injertos autólogos de cortical externa de calota craneana y de cresta ilíaca particulada, consiguiendo un volumen aceptable con ambos para garantizar una adecuada regeneración ósea, y en la fase de rehabilitación oral conseguir la instalación de implantes dentales.

\section{Reporte de caso:}

Se reporta el caso de un paciente masculino de 14 años de edad con un tiempo de enfermedad de 8 meses, natural y procedente Cuzco, quién acude al Servicio de Cirugía Oral y Maxilofacial del Hospital Nacional Cayetano Heredia Lima Perú.

Al examen clínico extraoral

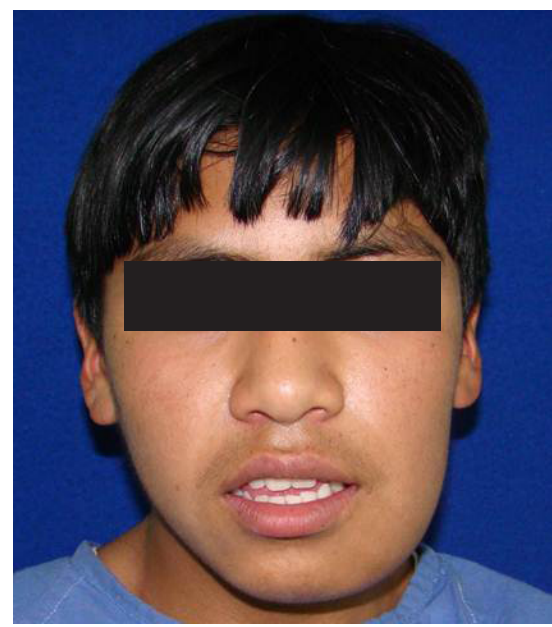

Fig. 1: Asimetría facial, tumefacción en región geniana baja izquierda con extensión a la región submaxilar ipsilateral.

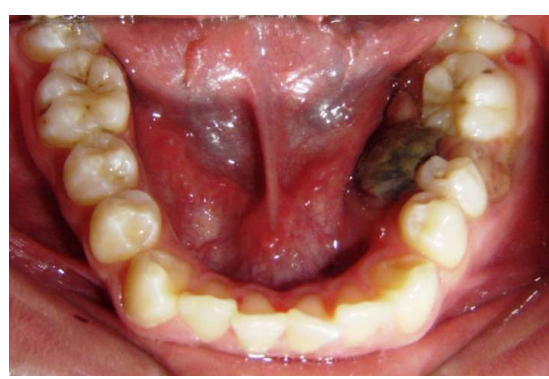

Fig. 2: Tumoración de $6 \times 4 \times 3 \mathrm{~cm}$ ubicado en el tercer cuadrante. 
presenta una asimetría facial en hemicara del lado izquierdo y una tumefacción en región geniana izquierda con extensión a la región submaxilar del mismo lado, de 8 x 6 x $5 \mathrm{~cm}$ de tamaño, consistencia y firme. Intraoralmente se observa aumento de volumen de $6 \times 4 \times 3 \mathrm{~cm}$ alrededor de piezas 35,36 y 37 con áreas de ulceración en la encía que la recubre, de coloración marrón parduzco, y de consistencia firme con áreas crepitantes, causando un borramiento de fondo de surco vestibular del tercer cuadrante.

Se indicó exámenes imaginológicas: Tomografía Espiral Multicorte con Volumen Rendering, Radiografía Panorámica, Radiografía Postero-Anterior tipo Towne y Oclusal tipo Donovan.

Se procedió a realizar una biopsia mediante punzo-aspiración por la zona crepitante, obteniendo un líquido de coloración amarillenta que coaguló rápidamente en la jeringa, seguido se realizó una biopsia por incisión cuyo resultado anatomopatológico fue un Ameloblastoma Plexiforme.

Se planifica el tratamiento qui-

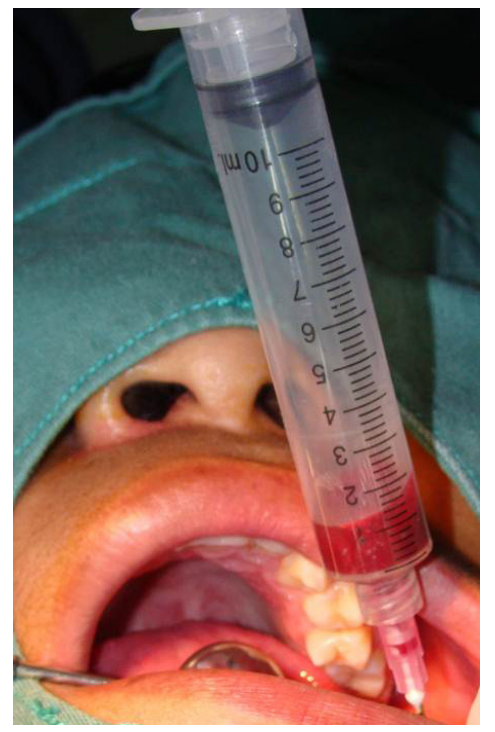

Fig. 3: Punzoaspiración, líquido citrino claro que se coagula rapidamente en jeringa.

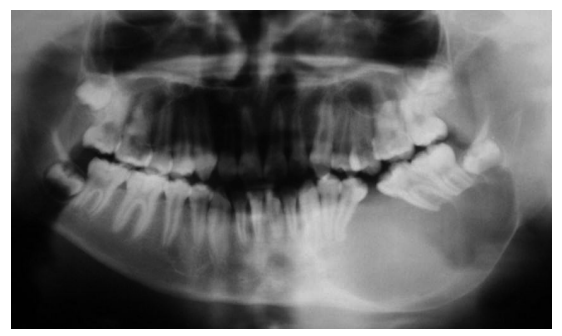

Fig. 4: Radiografía Panorámica inicial.

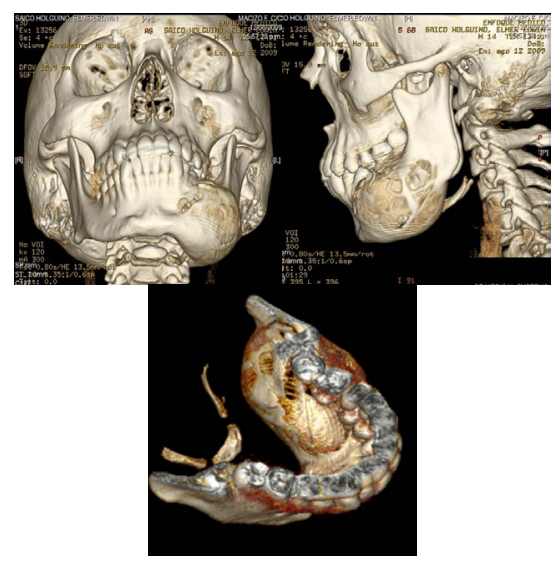

Fig. 5, 6, 7: TEM Volumen Rendering, extensión del tumor demostrando expansión de corticales.

rúrgico de la reconstrucción mandibular que consiste en resección quirúrgica parcial de la mandíbula de la zona afectada y reconstrucción inmediata, empleando la combinación de injertos autólogos libres de cortical externa de calota craneana y cresta ilíaca particulada, con fijación de una placa de reconstrucción del sistema 2.4.

En cuanto a la extensión de la resección mandibular, debemos de considerar el tamaño del tumor, la localización (maxilar superior, mandíbula), infiltración a tejidos blandos y compromiso de estructuras vecinas, basándonos en nuestra evaluación pre-operatoria y diagnóstico anatomopatológico que consideramos esencial en la terapéutica de este tipo de tumores.

El injerto se obtuvo de la cortical externa de la calota craneana y se planificó a través de una plantilla confeccionada con un dique de goma sobrepuesta a la radiografía cefalométrica lateral, con el cual se copió la extensión del tumor (incluyendo los márgenes de seguridad), y así mismo conocer la dimensión del injerto a utilizar.

Después de 04 meses de periodo postoperatorio se evalúa la formación ósea y se procede a colocar cuatro implantes endo-óseos diferidos.

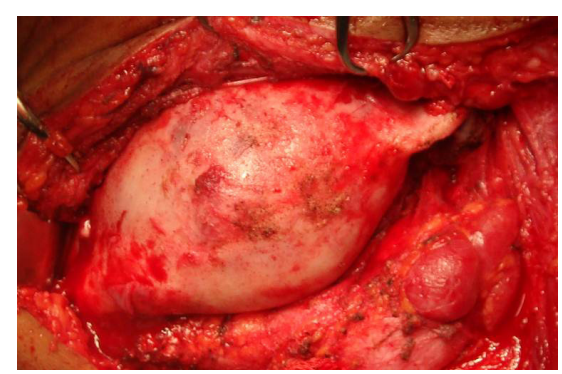

Fig. 8: Presentación del tumor delimitación de márgenes de seguridad.

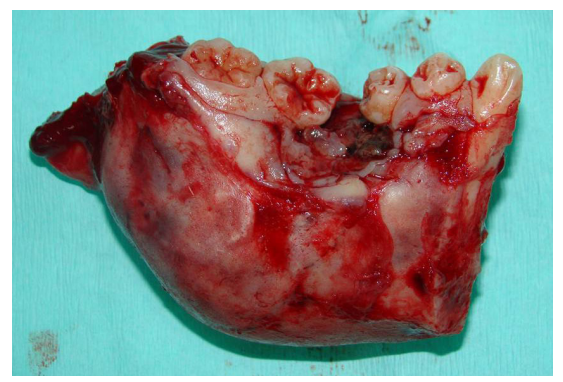

Fig. 9: Pieza Quirúrgica.

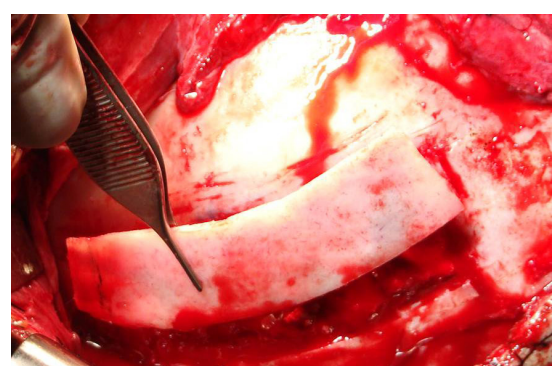

Fig. 10: Toma de injerto de cortical externa de calota craneana.

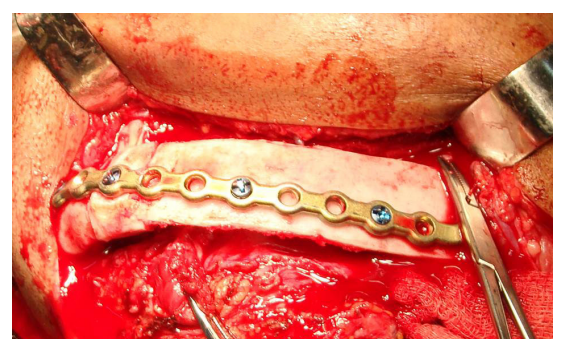

Fig. 11: Fijación rígida y estabilización de injertos óseos libres. 


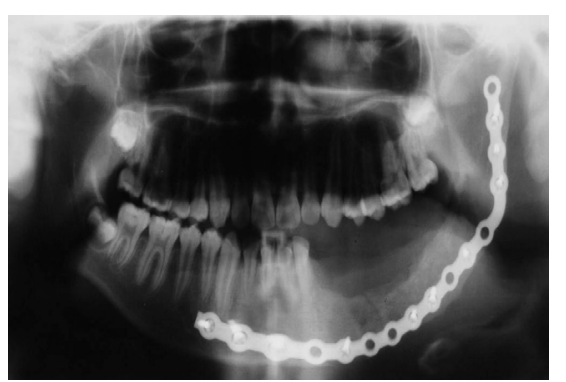

Fig. 12: Radiografía panorámica postoperatoria, placa de reconstrucción.

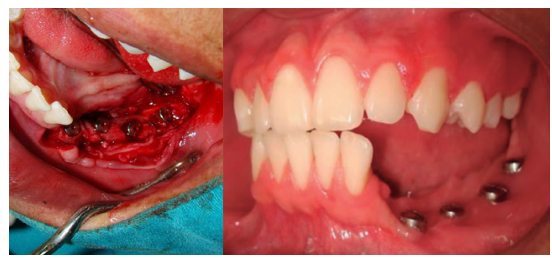

Fig. 13, 14: Primera y segunda fase de colocación de implantes.

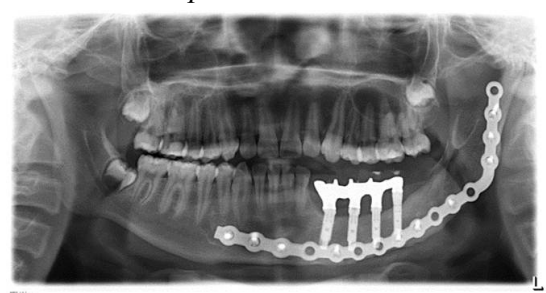

Fig. 15: Radiografía panorámica de control de instalación de implantes.

\section{Discusión:}

Es importante considerar en este tipo de lesiones que a pesar de ser tumores benignos tienen un comportamiento muy agresivo y que el potencial de recidiva es considerable; el tratamiento continúa siendo controversial, la literatura describe dos estrategias fundamentales de manejo:

Tratamiento conservador para las lesiones pequeñas, en dónde se diseña un abordaje menos agresivo.

Tratamiento agresivo en dónde la extirpación del tumor causará defectos considerables, lo que significa planificar un tratamiento reconstructivo, en el mismo tiempo acto operatorio.

Las tasas de recurrencia publicadas son altas y oscilan entre un $15 \%-25 \%$ luego de un tratamiento quirúrgico, pero se incrementan es- tas cifras mucho más en un rango aproximado de $75 \%-90 \%$ con un tratamiento conservador (8). El seguimiento de estos pacientes debe ser a largo plazo, ya que se han publicado cuadros de recidiva luego de 25 años a 30 años del tratamiento quirúrgico inicial.

Es necesario conocer la importancia funcional post-quirúrgica que acarrean las resecciones mandibulares, que guarda entre sus principales desventajas: problemas en el sistema masticatorio, foniátrico y deglución, de incompetencia labial y estéticas, que deben ser considerados en la planificación pre-quirúrgica.

Otra consideración a tomar en cuenta, es la selección de pacientes que serán sometidos a la resección quirúrgica con la técnica de reconstrucción mandibular complementaria, para determinar el tipo de injerto que se va a emplear. Carlson y Marx en 1996(9) aseguran, que el único protocolo que se debe utilizar en la planificación de la reconstrucción mandibular, es considerar la evaluación integral del paciente, y las ventajas y desventajas que le brindará el procedimiento quirúrgico; Desde 1966, aparecen publicaciones que describen las ventajas que ofrecen los injertos de calota craneal en la reconstrucción facial, dentro de las cuáles se describen: El dolor postoperatorio mínimo que ceden ante analgésicos (suaves) por vía oral, quienes proporcionan una disminución del dolor satisfactoria en el período postoperatorio inmediato(10). El uso de los injertos del hueso tomados de la calota craneal proporciona resultados fiables en el área de la cirugía reconstructiva maxilo y craneofacial, ya que la consistencia del hue- so craneal posee una cortical densa, una red haversiana rica que le permite revascularizarse rápidamente condicionando una reabsorción mínima. Indicando el empleo de éstos en casos de traumatismos maxilofaciales, defectos orbitarios, en el relleno de secuelas de osteotomías amplias en el macizo facial, en la reconstrucción esquelética de las deformidades dentofaciales y reconstrucciones mandibulares (11).

Al requerir una mayor cantidad de hueso en extensión, se requiere una incisión más amplia o el empleo de un abordaje tipo coronal, lo que afectará definitivamente el pronóstico de recuperación del paciente en cuanto al período de hospitalización. Se pueden producir daños neurológicos al momento de la toma del injerto, como hematomas subdurales, por lo que actualmente se recomienda el uso de sierras oscilantes y reciprocantes con instrumental mecánico. Hemorragias intra y post-operatorias se describen como consecuencia de una laceración de la arteria meníngea posterior, es uno de los vasos más afectados (10).

La primera tentativa de utilizar la calota en la reconstrucción craneal fue hecha desde 1670 por Van Meekren, quién reparó un defecto craneal de un soldado ruso con el hueso de un perro. Ollier 1867 acentuó el papel del periostio en la regeneración del hueso, en 1878 Macewen, según se informa, trasplantó con éxito un hueso alogénico en humanos, en 1891 Bardenheur fue el primero en realizar un injerto de hueso autólogo a la mandíbula. En 1908. El primer autotransplante osteoperióstico del cráneo fresco (hueso) fue realizado por Bunge en 1903; posteriormente, Sicard, Dambrin y Rogelio, entre 1917 y 1919, utilizaron el cráneo de un cadáver para 
realizar craneoplastias(12).

Una de las técnicas más usadas es la descrita por Tessier publicada en 1971, en dónde indica la osteotomía de la cortical externa de la calota craneana del área Parietal, reportando dentro de las ventajas: larga cantidad de cortical ósea, amplia tasa de éxito de oseointegración de implantes colocados sobre los injertos, escasas complicaciones de la zona donadora, mejor manejo de complicaciones, de las deshicencias post-operatorias y bajas tasas de reabsorción del injerto $(16,2 \%$ en un período postoperatorio de 6 meses y de 19,2\% en un período post-operatorio de 1 año). Así mismo, como potenciales complicaciones o desventajas; alopecia, seromas, hematomas infectados, exposición de la duramadre, hemorragia intracraneal, meningitis, injurias cerebrales y salida de líquido cefalorraquídeo (13).

Es importante también considerar la importancia del uso del injerto de cresta ilíaca particulada contenida por el flanco lateral de la calota craneal. La rápida accesibilidad demostrada en nuestra institución por parte del equipo de traumatología en conjunto con el equipo de cirugía maxilofacial ante la toma del injerto de cresta ilíaca anterior, las complicaciones son nulas en sala de operaciones, eso hace que consideremos como un método seguro y efectivo que puede emplearse en estos procedimientos de Reconstrucción Mandibular. Freilich y Sandor (14) consideran el Gold Standard de los Injertos Autólogos, ya que cantidades considerables de hueso cortico-medular son obtenidas de la Cresta Ilíaca anterior, la cual se partícula a manera de chips para ubicarlo en la parte central de la zona intervenida destinada a la reconstrucción.

En la actualidad, se considera que el injerto microvascularizado de peroné es la mejor opción de tratamiento para las reconstrucciones mandibulares de defectos óseos mayores de $5 \mathrm{~cm}$ que resultan de traumatismos, infecciones o resecciones tumorales. La discrepancia de altura entre el injerto de peroné y el reborde alveolar de la hemimandíbula dentada contralateral supone una dificultad importante para que ésta se lleve a cabo satisfactoriamente. Esta discrepancia de altura conduciría a una sobrecarga de fuerzas sobre los implantes, lo cual afectaría a la supervivencia de los mismos a largo plazo, por lo que el autor sugiere la distracción osteogénica vertical del injerto de peroné (15) Mehrotra y cols. coinciden que los injertos avascularizados muestran amplias ventajas en cuanto a la reconstrucción de forma facial, contorno, forma del arco y altura del hueso alveolar mandibular para la posterior colocación de implantes. Debemos de tener en cuenta que el empleo del injerto autólogo de cresta ilíaca se utiliza en pacientes en los que no sospechamos la recurrencia de la lesión (16).

Es importante tener en cuenta que esta pérdida de minerales es normal, y para anticipar los efectos, el injerto no se debe esperar para soportar cargas importantes sin la ayuda de la fijación interna adecuada durante el esta fase de constitución. Después de esta ola de actividad de reabsorción, comienza, la nueva formación de hueso en unos meses y poco a poco vuelve a la normalidad al recibir fuerzas mecánicas (17).
Los requerimientos óseos para una reconstrucción óptima y funcional, incluyen una adecuada longitud, cantidad, calidad e irrigación ósea. La rehabilitación dental de pacientes que han sido sometidos a mandibulectomías representan un desafío muy grande para los rehabilitadores orales al planificar la colocación de implantes dentales sobre los mismos. Urken fue el primero en reportar esta técnica de rehabilitación oral con implantes dentales colocados inmediatamente sobre injertos microvascularizados en 9 pacientes sometidos a reconstrucción. En cuanto a la evaluación de la oseointegración resulta interesante la tasa de éxito $(100 \%)$ reportada aún en mandíbulas sometidas a radiación y tratadas en conjunto con oxígeno hiperbárico, describiendo un seguimiento no menor de 5 años de los implantes sometidos a carga funcional (18).

La principal desventaja de este procedimiento es la considerable tasa de reabsorción que presentan este tipo de injertos, pero que al realizar el manejo con las Proteínas Morfogenéticas mejora considerablemente la tasa de éxito (19).

Una condición para el éxito del tratamiento es el retiro adecuado de la lesión tumoral con márgenes de seguridad indicados para así exponer el hueso sano y proceder a la reconstrucción inmediata de la zona afectada. Se utilizó la calota craneana como elemento de contención y recuperación anatómica, asimismo injerto particulado de cresta ilíaca para obtener un volumen óseo adecuado, que nos permita realizar una rehabilitación fija implantosoportada con resultados anatomo, funcionales y estéticos. 


\section{Conclusiones:}

El tratamiento realizado es una variante de todos los descritos en la literatura, que cubre las expectativas y necesidades quirúrgicas consideradas dentro de nuestros objetivos principales, y que permitió la integración rápida y efectiva del paciente a la sociedad.

Los tratamientos combinados de injertos óseos y prótesis implanto soportadas cumplen un objetivo bio-sicosocial, en pacientes con historia de tumores que requieren resecciones óseas amplias.

Con el empleo de esta técnica, se plantea el uso de la cortical externa de calota craneana como elemento de contención, estabilidad y recuperación anatómica inmediata, combinándolo con un injerto particulado de cresta ilíaca anterior para obtener un adecuado volumen óseo y confeccionar la rehabilitación con implantes dentales.

Hay que destacar la importancia y concientización del paciente en cuanto a los controles periódicos clínicos e imaginológicos rigurosos que dictaminarán el éxito a largo plazo de este tratamiento.

El caso fue tratado en forma multidisciplinaria e integralmente por los diferentes servicios de la Universidad Peruana Cayetano Heredia (Cirugía Oral y Maxilofacial, Radiología Oral y Maxilofacial e Implantología Oral).

\section{Agradecimientos:}

A los asistentes y residentes de los Servicios de Cirugía Oral y Maxilofacial e Implantolgía Oral de la U.P.C.H y del Hospital Nacional Cayetano Heredia, por el manejo social de este caso.

\section{Revisión Bibliográfica}

1. Zhang J, Gu Z, Jiang L, Zhao J, Tian M, Zhou J, et al. Ameloblastoma in children and adolescents. Br J Oral Maxillofac Surg. 2010 Oct:48(7):549-54.

2. Carini F, Riazzoli G, Vignoletti F, Baldoni M. Ameloblastoma plexiforme del maxilar: manejo quirúrgico y protético. A propósito de un caso. Av En Odontoestomatol. $2007 \mathrm{Feb}$;23(1):11-20.

3. Shetty RM, Rath S, Iyer V, Shetty S. Central Plexiform Ameloblastoma with Exophytic Growth: A Rare Case Report With Review Of Literature. Ann Essences Dent. 2010;2(2):106-11.

4. Singer SR, Mupparapu M, Philipone E. Cone beam computed tomography findings in a case of plexiform ameloblastoma. Quintessence Int Berl Ger 1985. 2009 Sep;40(8):627-30.

5. Carlson ER, Marx RE. The ameloblastoma: primary, curative surgical management. J Oral Maxillofac Surg Off J Am Assoc Oral Maxillofac Surg. 2006 Mar;64(3):484-94.

6. Sapp JP, Eversole LR, Wysocki GP. Patología oral y maxilofacial contemporánea. Elsevier España; 2005.

7. REGEZI J A. PATOLOGIA BUCAL McGraw-Hil Interamericana,Madrid; 2000.

8. Binali Ç, Fatma Ç, Oðuzhan A, Özkan M; Ameloblastom Pleksiform; Erciyes Týp Dergisi. Erciyes Medical Journal. 2009; 1:62-7.

9. Carlson ER, Marx RE. Mandibular reconstruction using cancellous cellular bone grafts. J Oral Maxillofac Surg. 54(7):889-97.

10.Harsha BC, Turvey TA, Powers SK. Use of autogenous cranial bone grafts in maxillofacial surgery: A preliminary report. J Oral Maxillofac Surg. 1986 Enero;44(1):11-5.

11.Ruiz RL, Turvey TA, Costello BJ, Tejera TJ. Cranial bone grafts: craniomaxillofacial applications and harvesting techniques. Atlas Oral Maxillofac Surg Clin North Am. 2005 Sep;13(2):127-37.

12.Artico M, Ferrante L, Pastore FS, Ramundo EO, Cantarelli D, Scopelliti D, et al. Bone autografting of the calvaria and craniofacial skeleton: historical background, surgical results in a series of 15 patients, and review of the literature. Surg Neurol. 2003 Jul;60(1):71-9.

13.Cuesta Gil M, Bucci T, Duarte Ruiz B, Riba García F. Reconstruction of the severely atrophic mandible using autologous calvarial bone graft: an " inverted sandwich" graft technique and dental rehabilitation with fixed implant-supported prostheses. J Cranio-Maxillo-Facial Surg Off Publ Eur Assoc CranioMaxillo-Facial Surg. 2010 Jul;38(5):379-84.

14.Freilich MM, Sándor GKB. Ambulatory in-office anterior iliac crest bone harvesting. Oral Surg Oral Med Oral Pathol Oral Radiol Endod. 2006 Mar;101(3):291-8.

15. Cho Lee GY, Naval Gías L, Rodríguez Campo FJ, Muñoz Guerra MF, Sastre Pérez J, González García R, et al. Distracción osteogénica vertical de injerto microvascularizado de peroné para reconstrucción mandibular y optimización de la rehabilitación implantológica. Rev Española Cirugía Oral Maxilofac. 2010 Abril;32(2):81-7.

16.Mehrotra D, Kumar S, Pradhan R. Mandibular reconstruction 
after resection of benign tumours using non-vascularised methods in a series of patients that did not undergo radiotherapy. Oral Surg. 2009;2(1):10-8.

17.Friedlaender GE. Bone grafts. The basic science rationale for clinical applications.
J Bone Joint Surg Am. 1987 Jun;69(5):786-90.

18.Khatami AH, Toljanic JA, Kleinman A. Mandibular reconstruction with vascularized fibula flap and osseointegrated implants: a clinical report. J Oral Implantol. 2010;36(5):385-90.
19.Abdel-Haleem AK, Nouby $\mathrm{R}$, Taghian M. The use of the rib grafts in head and neck reconstruction. Egypt J Ear Nose Throat Allied Sci. 2011 Jul;12(2):89-98. 